\title{
Body mass index, weight gain during pregnancy, and birth outcomes in women with self-report eating disorder traits
}

\author{
Brenda Beverly Broussard* \\ Seattle University College of Nursing, United States
}

Received: October 21, 2016

Accepted: January 6, 2017

Online Published: January 19, 2017

DOI: $10.5430 / \mathrm{cns} . v 5 \mathrm{n} 1 \mathrm{p} 29$

URL: http://dx.doi.org/10.5430/cns.v5n1p29

\begin{abstract}
Objective: To investigate body mass index (BMI), weight gain during pregnancy, and birth outcomes among postpartum women with self-report psychological traits associated with eating disorders.

Methods: A retrospective descriptive design was used to collect self-report eating disorder traits among a convenience sample of 54 postpartum women 19 to 43 years of age from a large hospital in the Pacific Northwest. Participants voluntarily completed a demographic form and Eating Disorder Inventory-3 (EDI-3) prior to discharge. EDI-3 risk scale scores (EDRS) were used to identify probable eating disorders. Medical records were reviewed for eating disorder history, BMI, weight gain during pregnancy, and birth outcomes.

Results: Fifteen $(27.8 \%)$ participants EDRS suggested the presence of an eating disorder, while one of the 54 medical records $(1.85 \%)$ indicated a previous history. Women with self-report psychological traits associated with eating disorders and complete prenatal records inclusive of height and preconception weight $(\mathrm{n}=10)$ had a mean BMI of 29.6 (clinically overweight/pre-obese). Of this subgroup, $60 \%(n=6)$ gained more weight during pregnancy than recommended guidelines. Nearly $67 \%(n=10)$ of the 15 participants had a cesarean section; two infants (13.3\%) were born preterm and one (6.7\%) was post-term. One infant (6.7\%) was born with a neural tube defect.

Conclusions: An overweight/pre-obese prenatal BMI and excessive weight gain during pregnancy may be indicative of an eating disorder. Women with self-report traits associated with eating disorders may be at risk for untoward pregnancy outcomes.
\end{abstract}

Key Words: Eating disorder, Body mass index, Weight gain, Pregnancy

\section{INTRODUCTION}

Pre-conception body mass index (BMI) and weight gain over the course of pregnancy are standard measures used by healthcare providers in determining maternal and fetal health and wellbeing. In 2009, the Institute of Medicine (IOM) ${ }^{[1]}$ revised the guidelines for adequate weight gain in accordance with BMI categories set forth by the World Health Organization (WHO). ${ }^{[2]}$ The recent adjustment allowed for greater flexibility in tailoring prenatal care to the unique, multifaceted nutritional needs of contemporary women. Moreover, the new guidelines are aimed at addressing the growing problem of obesity worldwide and the associated health risks for both mother and baby. ${ }^{[3,4]}$ BMI classifications and pregnancy weight gain recommendations are summarized in Table 1.

Despite having readily available and clear guidelines, nutri-

*Correspondence: Brenda Beverly Broussard; Email: broussab@ seattleu.edu; Address: Seattle University College of Nursing, United States. 
tional advice continues to be a challenge for health care providers and patients. A study that examined prenatal weight gain counseling ${ }^{[5]}$ found that only $41.7 \%$ of participants $(\mathrm{N}=401)$ reported that they received advice on how much weight they should gain. Of these women, $85 \%$ were given correct information within $\mathrm{IOM}^{[1]}$ standards. Participants who were classified as overweight or obese $(n=198)$ were more likely to receive advice to gain weight above $\mathrm{IOM}^{[1]}$ recommendations than women who were of normal weight $(\mathrm{n}=203)$. Likewise, Ferrari and Siega-Riz ${ }^{[6]}$ found that just $51 \%$ of women in their study $(\mathrm{N}=1,454)$ reported that a health care provider gave them advice on weight gain. In addition, $78 \%$ of the participants indicated that they gained more weight than was recommended.

Table 1. $\mathrm{WHO}^{[2]} \mathrm{BMI}$ categories and $\mathrm{IOM}^{[1]}$ recommended weight gain during pregnancy

\begin{tabular}{|c|c|}
\hline $\begin{array}{l}\text { WHO BMI Category and } \\
\text { Score }^{*}\left(B M I=\mathrm{kg} / \mathrm{m}^{2}\right)^{* *}\end{array}$ & $\begin{array}{l}\text { IOM }^{[1]} \text { Recommended Weight } \\
\text { Gain During Pregnancy**** }(\mathrm{lbs} / \mathrm{kg})\end{array}$ \\
\hline Underweight $<18.5^{*}$ & $28-40 / 12.5-18^{* * *}$ \\
\hline Normal Weight $18.5-24.9^{*}$ & $25-35 / 11.5-16^{* * *}$ \\
\hline \multicolumn{2}{|l|}{ Overweight/Pre-obese $\geq 25$} \\
\hline - Pre-obese $25-29.9^{* *}$ & $15-25 / 7-11.5^{* * * *}$ \\
\hline - Class I Obese $30-34.9^{* * *}$ & $11-20 / 5-9^{\text {**** }}$ \\
\hline - Class II Obese 35-39.9** & $11-20 / 5-9^{* * * *}$ \\
\hline - Class III Obese $\geq 40^{* *}$ & $11-20 / 5-9^{* * *}$ \\
\hline
\end{tabular}

Note. ${ }^{*}$ Adapted from World Health Organization (WHO) ${ }^{[2]}$ Technical Report Series 894 ; $^{* *}$ Body mass index (BMI) is calculated by dividing weight by $\mathrm{kg} / \mathrm{m}^{2}{ }^{* * * *}$ The Institute of Medicine (IOM) ${ }^{[1]}$ recommended weight gain during pregnancy in pounds/kilograms (lbs/kg)

Maternal overweight and obesity are global issues that also affect low and middle-income countries. Between 1980 and 2008, maternal BMI classifications of overweight $\left(>25 \mathrm{~kg} / \mathrm{m}^{2}\right)$ and obese $\left(>30 \mathrm{~kg} / \mathrm{m}^{2}\right)$ have risen substantially in all regions of the world including Africa, Asia, Oceania, Europe, the Americas, and the Caribbean. ${ }^{[3]}$ Current research looking at BMI and weight gain suggests an increase in untoward effects for women and/or infants when either under or overweight prior to pregnancy onset; or if weight gain fell outside of the parameters of "adequate" for the woman's initial BMI. ${ }^{[3,7-9]}$ Women identified as obese or gain more than $\mathrm{IOM}^{[1]}$ recommendations experience greater rates of cesarean delivery, large-for-gestational age (LGA) infants, and longer postpartum hospitalizations; while women who are underweight or do not gain a sufficient amount of weight are at a greater risk for preterm birth and infant mortality. ${ }^{[3,9]}$ A large-scale retrospective study that looked at maternal obesity found that when compared to women with a normal BMI (BMI 20-24.9, $\mathrm{n}=9,171$ ), women who began pregnancy with a BMI > 30 (obese, $\mathrm{n}=3,744$ ) had higher occurrences of hypertension, gestational diabetes, induction of labor or cesarean birth; and infants born LGA, or requiring admission to specialized neonatal care nurseries. ${ }^{[7]}$

Similarly, a large population-based cohort study $(n=99,403)$ in the United Kingdom (UK) examined the prevalence of perinatal obesity and outcome measures of cesarean birth, preterm birth, neonatal death, stillbirth, macrosomia, smallfor-gestational age (SGA), and LGA. Pre-conception BMI and weight categories were as follows: $2.88 \%(n=2,865)$ were underweight while $50.57 \%(n=50,488)$ were classified as normal weight, $28.43 \%(n=28,258)$ were overweight/preobese, $16.09 \%(\mathrm{n}=15,995)$ were listed as obese $\left(\mathrm{WHO}^{[2]}\right.$ class I and II obesity combined), and $1.85 \%(n=1,837)$ of participants were identified as morbidly obese $\left(\mathrm{WHO}^{[2]}\right.$ class III obesity). Cesarean births, LGA, and macrosomia were found to rise as BMI increased, with women categorized as morbidly obese having the greatest risk for these three outcomes. In addition, women who were overweight, obese, and morbidly obese had a higher risk of post-term delivery than women who had a normal weight. Finally, women who were underweight had a significant risk of preterm birth ( $<37$ weeks gestation) or delivering a baby that was $<33$ weeks gestation. ${ }^{[8]}$

Overall, research into maternal overweight or underweight suggests that women in these two groupings are at a greater risk for adverse pregnancy and neonatal outcomes. Presently, there is an increasing body of literature examining birth outcomes in women with a current or previous history of an eating disorder with similar findings. Eating disorders are considered a group of psychological conditions that encompass abnormal eating behaviors and ways of thinking about food and one's self or body. ${ }^{[10]}$ The three major disorders, anorexia nervosa (AN), bulimia nervosa $(\mathrm{BN})$, and binge-eating disorder (BED). A longitudinal study on pregnancy in the UK looked at birth outcomes among women ( $n=12,254$ ) who self-identified according to one of the following: (1) those who have ever had AN ( $n=171)$; or (2) BN ( $n=199)$; (3) women who ever had both $\mathrm{AN}$ and BN ( $n=82$ ), (4) those who have had a mental health or psychiatric disorder such as clinical depression $(n=1,166)$, and women that did not identify with any of the categories who served as controls $(n=10,636)$. Rates of preterm birth, deliveries occurring at less than 37 weeks gestation, determined by medical record data were as follows: AN 6.5\%, $\mathrm{BN} 5.0 \%, \mathrm{AN}$ and $\mathrm{BN} 4.9 \%$, mental health or psychiatric disorder $5.8 \%$, and controls $4.8 \%$. Although not statistically significant, women with a history of an eating disorder or psychiatric conditions had more preterm births than the control group. ${ }^{[11]}$

The Norwegian Mother and Child Cohort Study ${ }^{[12]}$ examined a variety of factors during and post-pregnancy, one 
of which was weight gain. Women who participated in the large population-based investigation $(\mathrm{N}=35,148)$ were categorized into one of the following groups: (1) AN, (2) BN, (3) eating disorder not otherwise specified purging type (EDNOS-P), (4) Binge Eating Disorder (BED), and (5) no eating disorder (control group). The determination of eating disorder was achieved through a self-report questionnaire developed by the researchers in accordance with diagnostic criteria set forth by the Diagnostic and Statistical Manual of Mental Disorders $4^{\text {th }}$ edition (DSM-IV). ${ }^{[13]}$ The majority of participants reported having no eating disorder before or during pregnancy $(\mathrm{n}=32,311 ; 94 \%)$ followed by $\operatorname{BED}(\mathrm{n}=1,737 ; 5.1 \%), \mathrm{BN}(\mathrm{n}=275 ; 0.8 \%), \mathrm{AN}(\mathrm{n}=32$; $0.1 \%)$, and EDNOS-P $(\mathrm{n}=31 ; 0.1 \%)$. Mean pre-conception $\mathrm{BMI}$ based on $\mathrm{WHO}^{[2]}$ categories for each of the groups were 24 for no eating disorder/control group (normal weight), 26 for BED (pre-obese), 24.3 for BN (normal weight), 18.1 for AN (underweight), and 24 for EDNOS-P (normal weight). Self-report total weight gain during pregnancy among the five groups was classified as inadequate, adequate, or excessive. The AN group had $21.9 \%$ gain an inadequate amount, $28.1 \%$ adequate, and $50 \%$ excessively. Women in the BN group had $20 \%$ gain inadequately, $20.4 \%$ adequately and $59.7 \%$ excessively. The EDNOS-P group showed $19.4 \%$ inadequate weight gain, $25.8 \%$ adequate gain, and $50 \%$ excessive. For the BED group, $17.6 \%$ gained an inadequate amount, $17.1 \%$ adequate, and $65.3 \%$ gained excessively. Lastly, women in the control group had $24 \%$ gain inadequately, $25 \%$ adequate weight gain, and $50 \%$ gained excessively. When compared to the control group, the findings suggest that women in the $\mathrm{BN}$ and BED group gained significantly more weight during pregnancy, and tended to gain in the excessively category at a higher percentage than the other groups.

Pre-pregnancy nutrition and BMI, as well as weight gain during pregnancy are a few of the essential components for a healthy pregnancy. Yet very few studies have looked at self-report traits associated with eating disorders and preconception BMI and weight gain during pregnancy. The aim of this article is to report findings from a pilot study on BMI, weight gain during pregnancy, and pregnancy outcomes among postpartum women with self-report traits associated with eating disorders.

\section{Methods}

\subsection{Design}

A retrospective descriptive design was used to collect selfreport eating disorder traits, preconception BMI, weight gain during pregnancy, and pregnancy outcomes among a convenience sample of postpartum women from a large hospital in the Pacific Northwest. Women who were 18 years or older, delivered a live birth at or greater than 25 weeks gestation, could understand and read English, and were able to complete the study forms were invited to participate. Those who volunteered to participate completed a demographic form and the Eating Disorder Inventory-3 (EDI-3) prior to discharge. EDI-3 risk scale scores (EDRS) were used to identify probable eating disorders. Medical records were reviewed for eating disorder history, BMI, weight gain during pregnancy, and pregnancy outcomes.

Hospital charts and change of shift report sheets were used to identify potential participants. Those who met criteria and gave oral consent were given a packet that contained a demographic form, EDI-3, two letters of consent, a pencil, and an envelope to place completed study materials. Information on the consent form included contacting their healthcare provider in the event that the EDI-3 questions caused concerns about disordered eating. Completed study packets were collected by the postpartum nurse and placed in a locked metal box at the nurses' station for collection by the research team.

One hundred postpartum women accepted invitation to participate and sixty declined. One study packet was mailed to the primary investigator's office and fifty-five packets were placed in the study box before discharge from the hospital $(\mathrm{n}=56)$. Two of the packets in the study box were blank. The return and completion rate was 54\%. The study was approved by the institutional review boards for the protection of human subjects from the hospital where data were collected and the university.

\subsection{Participants}

The fifty-four women who participated in the study were 19 to 43 years of age $($ mean $=32.1+5.2$ yrs. $)$. The majority identified as white $(75.9 \%)$ followed by Asian $(9.25 \%)$ and more than one ethnicity $(5.6 \%)$. The remainder of participants' ethnicity was one Hispanic $(1.85 \%)$, one Pacific Islander $(1.85 \%)$, one Alaska Native $(1.85 \%)$, one Ethiopian $(1.85 \%)$, and one did not respond to the question. A large portion were married $(87 \%)$ followed by single with a male partner $(9.3 \%)$. One participant did not answer and one was single with no partner. Greater than half had graduated from college $(51.9 \%)$, and nearly a third had completed graduate school (27.7\%). Several participants had indicated they had some college (13\%). For the remaining women, one did not respond, one did not complete high school, one graduated from high school, and one completed trade school.

\subsection{Instruments}

A demographic form was used to collect background characteristics of the participants. The EDI-3, a 91-item self-report 
measurement of psychological traits associated with AN, BN, and EDNOS, was used to measure probable eating disorders and is consistent with DSM-IV-TR diagnostic criteria. ${ }^{[14]}$ Using a six-point forced-choice format, respondents answer to items as "always", "usually", "often”, "sometimes", "rarely", or "never". Examples of EDI-3 items include: "I eat sweets and carbohydrates without feeling nervous", "I eat when I am upset", "I stuff myself with food", and "I trust others". Reliability for the original Eating Disorder Inventory risk scales range between 0.80 and 0.92 for eating disordered group and 0.83 to 0.93 for female comparison group. Scoring a qualitative rating of "typical clinical" or "elevated clinical" on one or more risk scale is considered rare among non-clinical groups and suggests the presence of an eating disorder. The three risk scales are drive for thinness (DT), bulimia (B), and body dissatisfaction (BD). A score of typical or elevated clinical on one or more EDRS was used to identify participants with psychological traits associated with eating disorders. Medical records were reviewed for a documented history of eating disorders.

\subsection{Data analysis}

EDI-3 item responses were entered into the EDI-3 Scoring Software Program computer system to tabulate EDRS. Participants' BMI was calculated using the standard formula: $\mathrm{BMI}=\mathrm{kg} / \mathrm{m}^{2}$. Preconception weight, weight gain during pregnancy, BMI, demographic data, EDRS scores classified as typical or elevated clinical, eating disorder history from medical records, and pregnancy outcomes (delivery type, gestational age, fetal anomalies, and newborn length and weight) were entered into the Statistical Package for Social Sciences (SPSS; version 17.0) and coded to maintain confidentiality. Descriptive statistics were used to obtain average BMI, weight gain during pregnancy, and pregnancy outcomes for participants' with one or more EDRS score that was typical or elevated clinical.

\section{RESULTS}

Fifteen women $(27.8 \%)$ had a score of typical or elevated clinical on one or more EDRS suggesting the presence of an eating disorder, and were as follows: 14 participants $(93.3 \%)$ had typical clinical scores ([BD, $\mathrm{n}=7,46.6 \%$ ], [B, $\mathrm{n}=3$, $20 \%$ ], [B and BD, $\mathrm{n}=3,20 \%$ ], [B, BD, and DT, $\mathrm{n}=1$, $6.7 \%]$ ), and one $(6.7 \%)$ had an elevated clinical score on BD. One of the fifteen participants' medical records indicated a past history of an eating disorder $(6.7 \%$ of women with self-report traits associated with eating disorders; $1.85 \%$ of the sample size of 54). Demographic characteristics of these women reflected that of the larger sample described above.

Pre-pregnancy BMI scores among postpartum women with typical and elevated EDRS scores and medical records that included this data $(n=10)$ ranged from 21.1-46.8, with a mean of $29.6+7.2$ (overweight/pre-obese). For this group of women, most had a BMI classified as overweight/pre-obese $(40 \%, \mathrm{n}=4)$ followed by class I obese $(30 \%, \mathrm{n}=3)$, normal weight $(20 \%, \mathrm{n}=2)$, and class III obese $(10 \%, \mathrm{n}=1)$. Weight gain during pregnancy $(\mathrm{n}=15)$ ranged from $13-45$ pounds (lbs), with a mean of $30.2 \mathrm{lbs}(+8.9)$. Of the participants with medical records inclusive of BMI, $40 \%(n=4)$ gained within the suggested guidelines for their pre-conception BMI, and $60 \%(n=6)$ gained more weight than $\mathrm{IOM}^{[1]}$ recommendations. These participants had an average gain of $13.3(+16.1)$ pounds more, with a range of 3 to $46 \mathrm{lbs}$. BMI and weight gain during pregnancy is presented in Table 2.

Nearly $67 \%(n=10)$ of participants had a cesarean birth. Two infants $(13.3 \%)$ were born preterm $(n=1$ at 33 weeks; $\mathrm{n}=1$ at 36 weeks), and one $(6.7 \%)$ was post-term $(\mathrm{n}=1,42$ weeks). One baby (6.7\%) was born with a neural tube defect. Newborn birth weight and length were all appropriate for gestational age.

Table 2. Preconception BMI and weight gain during pregnancy $(\mathrm{n}=10)$

\begin{tabular}{llll}
\hline & $\begin{array}{l}\text { Preconception BMI } \\
\end{array}$ & WHO $^{[2]}$ Qualitative Classification n $(\%)$ & $\begin{array}{l}\text { Weight Gain During Pregnancy } \\
\left(\mathbf{I O M}^{[1]} \text { Recommended Range) } \mathbf{n}(\%)\right.\end{array}$ \\
\cline { 3 - 4 } & $2(20)$ & $2(100)$ & Over \\
\hline Normal Weight & $4(40)$ & $1(25)$ & $3(75)$ \\
Overweight/Pre-Obese & $1(33.3)$ & $2(66.7)$ \\
Class I Obese & $3(30)$ & & $1(100)$ \\
Class III Obese & $1(10)$ & & \\
\hline
\end{tabular}

Note. WHO: World Health Organization; BMI: Body mass index; IOM: The Institute of Medicine

\section{Discussion}

The majority of research examining BMI and weight gain during pregnancy has focused on women in terms of being under, normal, or overweight; or whether they had gained an adequate amount of weight according to $\mathrm{WHO}^{[2]}$ guidelines to support a healthy pregnancy. These examinations have underscored the importance of nutrition prior to and throughout the childbearing period. However, the studies typically do 
not address eating disorders as a potential underlying issue or possible contributing factor. To the researcher's knowledge, this is one of only a handful of investigations that looked at self-report psychological traits associated with eating disorders and pre-pregnant BMI, weight gain during pregnancy, and pregnancy outcomes.

Findings from the study participants with self-report traits associated with eating disorders are of clinical importance. The mean preconception BMI was 29.6 (overweight/pre-obese), and 8 out of $10(80 \%)$ of these women had classifications of overweight or higher. This result is somewhat similar to the Norwegian Cohort Study. ${ }^{[12]}$ However, women identified as having $\mathrm{AN}, \mathrm{BN}$, or EDNOS-P had a BMI that was either under or normal weight. The self-report traits associated with eating disorders as determined by the EDI- $3^{[14]}$ used in this pilot study, does not specify whether a person has AN, BN, EDNOS, or BED. The large percentage of women with a BMI of overweight or higher might suggest that they suffer with BED. If so, this could account for the unusually high proportion of women who had a preconception BMI categorized as overweight to class III obese.

With respect to $\mathrm{IOM}^{[1]}$ recommendations on weight gain during pregnancy based on $\mathrm{WHO}^{[2]} \mathrm{BMI}$ classifications, the majority of women gained more than the recommendations $(60 \%, \mathrm{n}=6)$ followed by within the specified guidelines $(40 \%, \mathrm{n}=4)$. None of the participants in this subgroup gained less than recommended. This finding is congruent with previous research that looked at weight gain in pregnancy among women classified as having an eating disorder, with those described as having $\mathrm{BED}$ and $\mathrm{BN}$ gaining significantly more weight. ${ }^{[12]}$ The women in this pilot study were not asked whether they received guidance on nutrition or information on how much weight they should gain during pregnancy. Nevertheless, this particular finding could suggest that these participants had $\mathrm{BED}, \mathrm{BN}$, or received advice to gain above $\mathrm{IOM}^{[1]}$ guidelines as was found in the study that looked at prenatal nutrition counseling and weight gain. [5]

The majority of participants had an adverse pregnancy outcome of cesarean birth. This finding is consistent with previous studies that suggest cesarean births are more likely among women who had a BMI that was overweight or higher. ${ }^{[7-9]}$ In addition, two (13.3\%) infants were born preterm. Research looking at eating disorders and pregnancy outcome found that women with an eating disorder were more likely to deliver a preterm baby. ${ }^{[11]}$ Finally, one $(6.7 \%)$ baby was born with a fetal anomaly of neural tube defect. None of the studies looking at BMI, weight gain, or eating disorders during pregnancy had this type of finding.

\subsection{Strengths and limitations}

Challenges associated with pilot studies typically center on generalizability of the findings due to small sample size and often, lack of diversity among participants. According to the DSM-V, ${ }^{[10]}$ estimated prevalence rates for the three main eating disorders $\mathrm{AN}, \mathrm{BN}$, and $\mathrm{BED}$, are $0.4 \%, 1 \%-1.5 \%$, and $1.6 \%$ respectively. When evaluated from this perspective, a sample size of $28.7 \%$ of participants $(n=15)$ might no longer be considered miniscule or too few to gain valuable insight into the problem. Another limitation is a response rate of $54 \%$. While this may be considered a good rate of return for survey data, important information may have been gained from the women who later chose not to complete their study packets. Even so, data from the 15 participants are essential in providing direction for further studies. A large-scale longitudinal study looking at the prevalence of self-report eating disorders, preconception BMI, nutrition advice, weight gain, and pregnancy outcome for both mother and baby may provide information that could potentially benefit maternal and child healthcare across the childbearing years.

\subsection{Clinical implications}

Women with an eating disorder do not necessarily present with a preconception BMI or weight gain that is vastly different from the general population. ${ }^{[12]}$ This coupled with the secretive nature of eating disorders makes it difficult for health care providers to identify women for whom this is a problem. Practitioners can use this information to alert themselves to the possibility that clients with a BMI of overweight or higher and/or gain more than recommended might have an eating disorder and warrants further assessment. Offering prenatal nutrition and weight gain counseling that is accurate and in line with current $\mathrm{IOM}^{[1]}$ standards could be used as the starting point for ongoing conversation with patients during prenatal visits.

\section{Conclusions}

In conclusion, a pre-pregnant BMI categorized as underweight or inadequate weight gain during pregnancy might not be the prevailing indicator of an eating disorder. Women classified as overweight and higher need to be regarded as potentially having an eating disorder. In addition, those who gain more than $\mathrm{IOM}^{[1]}$ recommendations should be considered as possibly having this issue as well. Women with self-report traits associated with eating disorders may be at risk for untoward pregnancy outcomes of cesarean section and preterm birth. Nurses and healthcare providers should assess for eating disorders throughout pregnancy regardless of past medical history, pre-pregnant BMI, or adequate weight gain. 


\section{CONFLicts OF INTEREST Disclosure}

No conflict of interest has been declared by the author.

\section{REFERENCES}

[1] Institute of Medicine. Weight gain during pregnancy: reexamining the guidelines. 2009. Available from: http: //www.nationalacademies.org/hmd/ /media/Fi les/Report\%20Files/2009/Weight-Gain-During-Pregnan cy-Reexamining-the-Guidelines/Report $\% 20 \mathrm{Brief} \% 20-\%$ 20Weight \%20Gain\%20During\%20Pregnancy.pdf

[2] World Health Organization. Obesity: preventing and managing the global epidemic. WHO technical series report 894. 2000, 2004. Available from: http://www. who.int/nutrition/publicatio ns/obesity/WHO_TRS_894/en/

[3] Black RE, Victora CG, Walker SP, et al. Maternal and child undernutrition and overweight in low-income and middle-income countries. Lancet. 2013; 382: 427-51. https : //doi .org/10.1016/S0 $140-6736$ (13) 60937-X

[4] World Health Organization. Obesity and overweight. 2014a. Available from: http://www.who.int/mediacentre/factsheets /fs311/en/

[5] Phelan S, Phipps MG, Abrams B, et al. Practitioner advice and gestational weight gain. J Women's Health. 2011; 20(4): 585-591. https://doi.org/10.1089/jwh. 2010.2316

[6] Ferrari RM, Siega-Riz AM. Provider advice about pregnancy weight gain and adequacy of weight gain. Matern Child Health J. 2013; 17: 256. https : //doi .org/10.1007/s10995-012-0969-z
[7] Joy S, Istwan N, Rhea D, et al. The impact of maternal obesity on the incidence of adverse pregnancy outcomes in high-risk term pregnancies. Am J Perinatol. 2009; 26(5): 345-9. https : //doi .org/10.1 055/s-0028-1110084

[8] Khashan AS, Kenny LC. The effects of maternal body mass index on pregnancy outcome. Eur J Epidemiol. 2009; 24: 697. https: //doi.org/10.1007/s10654-009-9375-2

[9] Mamun AA, Callaway LK, O'Callaghan MJ, et al. Associations of maternal and pre-pregnancy obesity and excess pregnancy weight gains with adverse pregnancy outcomes and length of hospital stay. BMC Pregnancy and Childbirth. 2011; 11: 1-9. https ://doi .or g/10.1186/1471-2393-11-62

[10] American Psychological Association. Diagnostic and Statistical Manual of Mental Disorders, 5th ed. Washington, DC; 2013.

[11] Micali N, Simonoff E, Treasure J. Risk of major adverse outcomes in women with eating disorders. BJP. 2007; 190: 255-259. https://doi.org/10.1192/bjp.bp.106.020768

[12] Siega-Riz AM, Von Holle A, Haugen M, et al. Gestational weight gain of women with eating disorders in the Norwegian Pregnancy Cohort. Int J Eat Disord. 2011; 44: 428-434. https ://doi.org/ 10.1002/eat. 20835

[13] American Psychological Association. Diagnostic and Statistical Manual of Mental Disorders, 4th ed. Washington, DC; 1994.

[14] Garner DM. Eating disorder inventory-3 professional manual. Lutz, Florida: Psychological Assessment Resources, Inc; 2004. 